\title{
Struktur des 2,2,4,4-Tetrakis(trifluormethyl)-1,3-diselenetans
}

Structure of 2,2,4,4-Tetrakis(trifluoromethyl)-1,3-diselenetane

Gerd Rabe, Klaus Keller, Herbert W. Roesky*, Richard J. Lagow, Frank Pauer und Dietmar Stalke Institut für Anorganische Chemie der Universität Göttingen,

Tammannstraße 4, D-3400 Göttingen

Herrn Prof. Dr. Dr.h.c. Ekkehard Fluck zum 60. Geburtstag gewidmet

Z. Naturforsch. 46b, 157-160 (1991); eingegangen am 6. August 1990

Hexafluoroselenoacetone, X-Ray

The reaction of hexafluoroacetone with triphenylphosphane selenide proceeds with elimination of triphenylphosphane oxide to yield 2,2,4,4-tetrakis(trifluormethyl)-1,3-diselenetane (1). Crystals of 1 that are suitable for X-ray crystal structure analysis have been obtained by recrystallization from hexane.

\section{Einleitung}

Die Chemie des Hexafluoracetons (HFA) ist eingehend untersucht worden [1]. Das homologe Hexafluorthioaceton (HFTA), welches dimer vorliegt, ist vermehrt in den Blickpunkt des Interesses [2] gerückt, seitdem es gut zugänglich ist [3].

Das nächst höhere Homologe, das dimere $\mathrm{He}$ xafluorselenoaceton (2,2,4,4-Tetrakis(trifluormethyl)-1,3-diselenetan) (1), ist hingegen lediglich zweimal in der Literatur beschrieben worden. Zum erstenmal wurde Verbindung $\mathbf{1}$ in einer Ausbeute von $6,4 \%$ als eines der Produkte der Reaktion von Hexafluorpropen und Selen in Gegenwart von Antimonpentafluorid erwähnt [4], jedoch ohne Angabe eines Schmelzpunkts oder weitergehende Charakterisierung außer einem Massenspektrum.

1980 berichtete Raasch [5] von einer gezielten Synthese von 1 mit einer Ausbeute von 73\% durch fünfstündiges Erhitzen von HFA und Triphenylphosphanselenid auf $150{ }^{\circ} \mathrm{C}$.

$$
\begin{aligned}
& \left(\mathrm{CF}_{3}\right)_{2} \mathrm{C}=0 \stackrel{\mathrm{Ph}_{3} \mathrm{P}=\mathrm{Se}}{\longrightarrow}\left[\begin{array}{cc}
\left(\mathrm{CF}_{3}\right)_{2}-\mathrm{C}_{\mathrm{C}}-\mathrm{Se} \stackrel{\oplus}{\mathrm{PPh}} \\
0
\end{array}\right] \\
& -\left[\left(\mathrm{CF}_{3}\right)_{2} \mathrm{C}_{-0}^{-\mathrm{Se}}-\mathrm{PPh}_{3}\right] \\
& \left.\underset{-0=\mathrm{PPh}_{3}}{-}\left[\left(\mathrm{CF}_{3}\right)_{2} \mathrm{C}=\mathrm{Se}\right] \longrightarrow \frac{1}{2}\left(\mathrm{CF}_{3}\right)_{2} \mathrm{C}_{-\mathrm{Se}}^{-\mathrm{Se}}-\mathrm{ClF}_{3}\right)_{2} \\
& 1
\end{aligned}
$$

\footnotetext{
* Sonderdruckanforderungen an Prof. Dr. H.W. Roesky.

Verlag der Zeitschrift für Naturforschung, D-7400 Tübingen 0932-0776/91/0200-0157/\$01.00/0
}

Als Reaktionsmechanismus scheint ein Sauerstoff-Selen-Austausch über einen intermediär gebildeten viergliedrigen Ring plausibel. Die Charakterisierung dieser Verbindung war jedoch bisher unvollständig. Folgereaktionen von 1 wurden nicht beschrieben. Wir waren daran interessiert, die Struktur dieser Verbindung $(2,2,4,4$-Tetrakis(trifluormethyl)-1,3-diselenetan) aufzuklären.

\section{Experimenteller Teil}

Alle Reaktionen werden unter Ausschluß von Luft und Feuchtigkeit durchgeführt. Die Lösungsmittel werden vor Gebrauch nach den üblichen Methoden getrocknet. IR-Spektrum: Bio-Rad FTS-7, 3240-SPC.-Massenspektrum: CH 5 Varian-MAT und Finnigan-MAT System 8230. NMR-Spektren: Bruker WP80 SY, AM 250.

\section{Darstellung des dimeren Hexafluorselenoacetons (1)}

In einem 11-Edelstahlzylinder mit Magnetrührer werden $50 \mathrm{~g}(147 \mathrm{mmol})$ Triphenylphosphanselenid und $25 \mathrm{~g}$ ( $150 \mathrm{mmol})$ HFA $48 \mathrm{~h}$ unter mechanischem Rühren auf $170^{\circ} \mathrm{C}$ erhitzt. Nach Abkühlen auf R.T. werden alle gasförmigen Bestandteile in einer Kühlfalle aufgefangen. Aus dem Rückstand erhält man durch Sublimation im statischen Vakuum bei $50{ }^{\circ} \mathrm{C} / 180$ Torr das Rohprodukt von 1 . Nach Umkristallisieren aus Hexan und wiederholter Sublimation im statischen Vakuum erhält man $6,7 \mathrm{~g}(20 \%) 1$ als blaßgelbe Kristalle. Schmp. $60{ }^{\circ} \mathrm{C}$.

IR (Nujol/KBr): 1245 sst, 1193 sst, 926 st, $907 \mathrm{st}, 737 \mathrm{sst}, 692 \mathrm{sst} \mathrm{cm}^{-1}$. Nur die starken und sehr starken Banden werden mitgeteilt.

${ }^{19} \mathrm{~F}-\mathrm{NMR}\left(\mathrm{CDCl}_{3}, \mathrm{CFCl}_{3}\right): \delta-70,1 \mathrm{ppm}$.

${ }^{13} \mathrm{C}-\mathrm{NMR}\left(\mathrm{CDCl}_{3}, \mathrm{TMS}\right): \delta 123,2\left(\mathrm{q}, J_{\mathrm{C}-\mathrm{F}}=280\right.$ $\mathrm{Hz}), 15,0\left(\mathrm{sp},{ }^{2} J_{\mathrm{C}-\mathrm{F}}=37 \mathrm{~Hz}, \underline{\mathrm{C}}-\mathrm{C}-\mathrm{F}\right) \mathrm{ppm}$.

${ }^{77} \mathrm{Se}-\mathrm{NMR}\left(\mathrm{CDCl}_{3}, \mathrm{Me}_{2} \mathrm{Se}\right): \delta 667,6 \mathrm{ppm}$. 


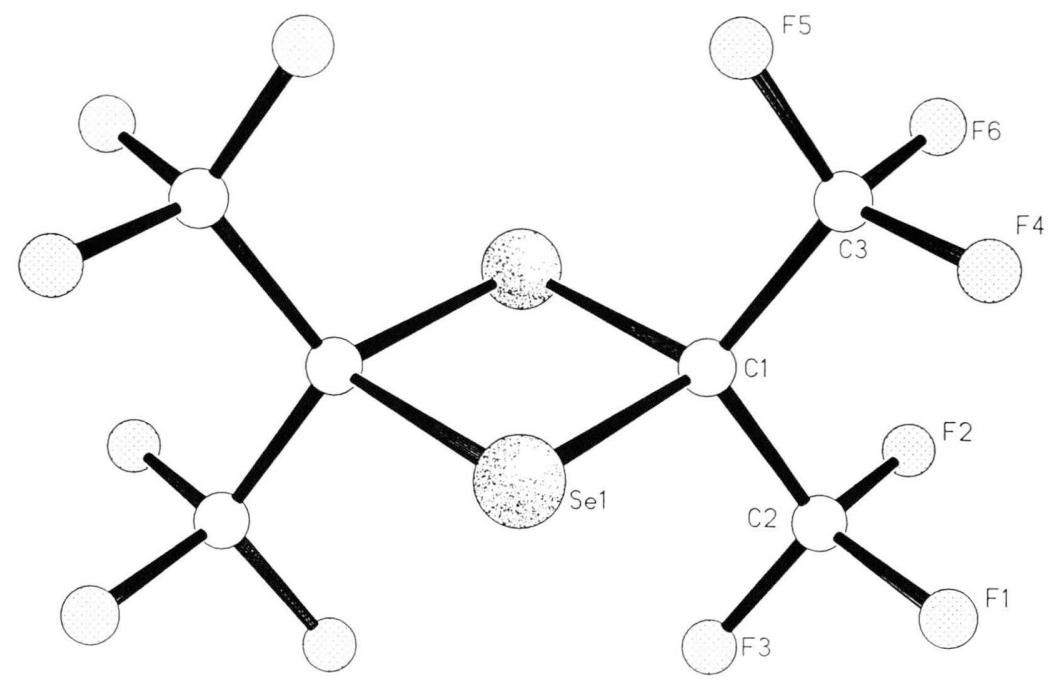

Abb. 1. Molekülstruktur von 1 im Kristall.

\section{Kristallstrukturanalyse von $\mathbf{1}$}

Geeignete Einkristalle von 1 werden durch Umkristallisieren aus Hexan erhalten. Die Kristalldaten sowie Angaben zu den Intensitätsmessungen und der Verfeinerung sind in Tab. I zusammengefaßt. Tab. II enthält die Bindungslängen und -winkel, Tab. III die Atomkoordinaten [6].

\section{Ergebnisse und Diskussion}

Die von Raasch beschriebene Synthese zur Darstellung von Verbindung 1 wurde von uns dahingehend variiert, daß anstelle eines verschlossenen Glasgefäßes als Reaktionsgefäß ein Edelstahlzylinder mit Rührmagnet verwendet wurde. Die Aufarbeitung nach Raasch durch Wasserdampfdestillation ersetzten wir durch Sublimation des Rohprodukts aus dem Reaktionsgemisch, da in unseren Händen Verbindung 1 durch Wasserdampfdestillation zersetzt wurde. Bei einer Reaktionsdauer von $48 \mathrm{~h}$ und einer Reaktionstemperatur von $170{ }^{\circ} \mathrm{C}$ konnten wir Verbindung 1 in Ausbeuten von etwa $20 \%$ isolieren.

Es sind bisher nur wenige Strukturuntersuchungen an 1,3-Diselenetanen durchgeführt worden. Das 2,2,4,4-Tetrafluor-1,3-diselenetan wurde durch Elektronenbeugung untersucht [7].

Einkristallröntgenstrukturanalysen wurden bisher lediglich am Beispiel des 2,2,4,4-Tetraacetyl1,3-diselenetans durchgeführt. Bemerkenswerterweise wird über diese Röntgenstrukturanalyse zweimal in der Literatur berichtet [8, 9]. Die jeweiligen Autoren finden unterschiedliche Raumgruppen.

Tab. I. Kristallographische Daten der Verbindung 1.

\begin{tabular}{|c|c|}
\hline Summenformel & $\mathrm{C}_{6} \mathrm{~F}_{12} \mathrm{Se}_{2}$ \\
\hline Molmasse & 458,0 \\
\hline Datensammlung bei $\mathrm{T}\left[{ }^{\circ} \mathrm{C}\right]$ & -120 \\
\hline Kristallgröße $\left[\mathrm{mm}^{3}\right]$ & $0,3 \times 0,5 \times 0,5$ \\
\hline Raumgruppe & $\mathrm{P} 2 / n$ \\
\hline$a[\mathrm{pm}]$ & $668,5(4)$ \\
\hline$b[\mathrm{pm}]$ & $981,3(6)$ \\
\hline$c[\mathrm{pm}]$ & $876,4(5)$ \\
\hline$\beta\left[{ }^{\circ}\right]$ & $108,03(4)$ \\
\hline Zellvolumen $\left[\mathrm{nm}^{3}\right]$ & 0,547 \\
\hline Formeleinheiten pro Zelle Z & 2 \\
\hline Berechn. Dichte $\varrho$ ber $\left[\mathrm{Mgm}^{-3}\right]$ & 2,782 \\
\hline Absorptionskoeffizient $\mu\left[\mathrm{mm}^{-1}\right]$ & 6,25 \\
\hline $\mathrm{F}(000)$ & 424 \\
\hline Gemessener $2 \theta$-Bereich $\left[{ }^{\circ}\right]$ & $7-56$ \\
\hline Anzahl der gemessenen Reflexe & 1821 \\
\hline Anzahl der unabhängigen Reflexe & 1266 \\
\hline Anzahl der beobachteten Reflexe & 1160 \\
\hline \multicolumn{2}{|l|}{ Übereinstimmung äquivalenter } \\
\hline Reflexe $\left(R_{\text {int }}\right)$ & 0,0244 \\
\hline $\mathrm{F}>\mathrm{p} \sigma(\mathrm{F}): \mathrm{p}=$ & 3 \\
\hline$R$ & 0,0532 \\
\hline $\mathrm{w} R$ & 0,0699 \\
\hline Goodness of fit & 1,7633 \\
\hline \multicolumn{2}{|l|}{ Gewichtsfaktor g } \\
\hline $\mathrm{w}=1 /\left[\sigma^{2}(\mathrm{~F})+\mathrm{abs}(\mathrm{g}) \mathrm{F}^{2}\right]$ & 0,0012 \\
\hline Verfeinerte Parameter & 92 \\
\hline \multicolumn{2}{|l|}{ Letzte Differenz-Fourier-Synthese } \\
\hline Größtes Maximum $\left[\mathrm{e}^{-} \mathrm{nm}^{-3}\right]$ & $12,5 \times 10^{2}$ \\
\hline Größtes Minimum $\left[\mathrm{e}^{-} \mathrm{nm}^{-3}\right]$ & $26,9 \times 10^{2}$ \\
\hline Extinktionskorrektur & ja \\
\hline
\end{tabular}




\begin{tabular}{lrll}
\hline $\operatorname{Se}(1)-C(1)$ & $197,1(4)$ & $\mathrm{Se}(1)-\mathrm{C}(1 \mathrm{~A})$ & $196,1(5)$ \\
$\mathrm{C}(1)-\mathrm{C}(2)$ & $154,0(7)$ & $\mathrm{C}(1)-\mathrm{C}(3)$ & $153,2(5)$ \\
$\mathrm{C}(2)-\mathrm{F}(1)$ & $133,2(6)$ & $\mathrm{C}(2)-\mathrm{F}(2)$ & $133,9(5)$ \\
$\mathrm{C}(2)-\mathrm{F}(3)$ & $131,9(5)$ & $\mathrm{C}(3)-\mathrm{F}(4)$ & $134,1(6)$ \\
$\mathrm{C}(3)-\mathrm{F}(5)$ & $132,7(6)$ & $\mathrm{C}(3)-\mathrm{F}(6)$ & $132,3(6)$ \\
$\mathrm{C}(1)-\mathrm{Se}(1)-\mathrm{C}(1 \mathrm{~A})$ & $83,6(2)$ & $\mathrm{Se}(1)-\mathrm{C}(1)-\mathrm{C}(2)$ & $111,6(3)$ \\
$\mathrm{Se}(1)-\mathrm{C}(1)-\mathrm{C}(3)$ & $111,6(3)$ & $\mathrm{C}(2)-\mathrm{C}(1)-\mathrm{C}(3)$ & $112,2(4)$ \\
$\mathrm{Se}(1)-\mathrm{C}(1)-\mathrm{Se}(1 \mathrm{~A})$ & $96,4(2)$ & $\mathrm{C}(2)-\mathrm{C}(1)-\mathrm{Se}(1 \mathrm{~A})$ & $112,3(3)$ \\
$\mathrm{C}(3)-\mathrm{C}(1)-\mathrm{Se}(1 \mathrm{~A})$ & $111,8(3)$ & $\mathrm{C}(1)-\mathrm{C}(2)-\mathrm{F}(1)$ & $111,3(4)$ \\
$\mathrm{C}(1)-\mathrm{C}(2)-\mathrm{F}(2)$ & $111,0(4)$ & $\mathrm{F}(1)-\mathrm{C}(2)-\mathrm{F}(2)$ & $107,4(4)$ \\
$\mathrm{C}(1)-\mathrm{C}(2)-\mathrm{F}(3)$ & $111,7(4)$ & $\mathrm{F}(1)-\mathrm{C}(2)-\mathrm{F}(3)$ & $107,5(4)$ \\
$\mathrm{F}(2)-\mathrm{C}(2)-\mathrm{F}(3)$ & $107,7(4)$ & $\mathrm{C}(1)-\mathrm{C}(3)-\mathrm{F}(4)$ & $111,1(4)$ \\
$\mathrm{C}(1)-\mathrm{C}(3)-\mathrm{F}(5)$ & $112,3(4)$ & $\mathrm{F}(4)-\mathrm{C}(3)-\mathrm{F}(5)$ & $106,7(4)$ \\
$\mathrm{C}(1)-\mathrm{C}(3)-\mathrm{F}(6)$ & $111,9(4)$ & $\mathrm{F}(4)-\mathrm{C}(3)-\mathrm{F}(6)$ & $107,1(4)$ \\
$\mathrm{F}(5)-\mathrm{C}(3)-\mathrm{F}(6)$ & $107,4(4)$ & & \\
\hline
\end{tabular}

Tab. II. Bindungslängen (pm) und -winkel $\left.{ }^{\circ}\right)$ von $\mathbf{1 .}$

Tab. III. Atomkoordinaten $\left(\times 10^{4}\right)$ und äquivalente isotrope Thermalparameter $\left(\mathrm{pm}^{2} \times 10^{-1}\right)$ von $\mathbf{1}$.

\begin{tabular}{lrrrr}
\hline & \multicolumn{1}{c}{$x$} & \multicolumn{1}{c}{$y$} & \multicolumn{1}{c}{$\mathrm{U}_{\text {eq }}$} \\
\hline $\mathrm{Se}(1)$ & $1151(1)$ & $1278(1)$ & $44(1)$ & $20(1)$ \\
$\mathrm{C}(1)$ & $161(6)$ & $-276(4)$ & $-1420(5)$ & $17(1)$ \\
$\mathrm{C}(2)$ & $2009(7)$ & $-1064(4)$ & $-1699(6)$ & $22(1)$ \\
$\mathrm{F}(1)$ & $3022(5)$ & $-307(3)$ & $-2486(4)$ & $37(1)$ \\
$\mathrm{F}(2)$ & $1332(5)$ & $-2188(3)$ & $-2573(4)$ & $39(1)$ \\
$\mathrm{F}(3)$ & $3394(5)$ & $-1444(3)$ & $-334(4)$ & $32(1)$ \\
$\mathrm{C}(3)$ & $-1462(7)$ & $167(5)$ & $-2997(5)$ & $25(1)$ \\
$\mathrm{F}(4)$ & $-583(5)$ & $971(3)$ & $-3850(4)$ & $34(1)$ \\
$\mathrm{F}(5)$ & $-3035(5)$ & $870(3)$ & $-2758(4)$ & $33(1)$ \\
$\mathrm{F}(6)$ & $-2291(5)$ & $-887(3)$ & $-3917(4)$ & $42(1)$ \\
\hline
\end{tabular}

* Äquivalente isotrope U berechnet als ein Drittel der Spur des orthogonalen $\mathrm{U}_{\mathrm{ij}}$-Tensors.

\begin{tabular}{|c|c|c|c|c|c|}
\hline & & $\mathrm{Se}-\mathrm{C} 1[\mathrm{pm}]$ & $\mathrm{Se}-\mathrm{C} 1 \mathrm{~A}[\mathrm{pm}]$ & $\mathrm{Se}-\mathrm{Se}[\mathrm{pm}]$ & $\mathrm{Se}-\mathrm{C}-\mathrm{Se}\left[{ }^{\circ}\right]$ \\
\hline 1 & & 19 & 196 & 293 & $96,4(2)$ \\
\hline $\mathrm{C}_{2} \mathrm{~F}_{4} \mathrm{Se}_{2}$ & [7] & $196,4(4)$ & $196,4(4)$ & $298,1(3)$ & $98,8(4)$ \\
\hline $\mathrm{C}_{10}^{2} \mathrm{H}_{12} \mathrm{O}_{4} \mathrm{Se}_{2}$ & [8] & $197,7(6)$ & $197,0(6)$ & $291,1(2)$ & $95,0(2)$ \\
\hline $\mathrm{C}_{10} \mathrm{H}_{12} \mathrm{O}_{4}^{4} \mathrm{Se}_{2}$ & [9] & $196,5(3)$ & $198,1(3)$ & $290,07(4)$ & $94,6(1)$ \\
\hline
\end{tabular}

Tab. IV. Vergleichende Übersicht der wichtigsten Bindungslängen und -winkel.

Einen Vergleich der wichtigsten Bindungslängen und -winkel von Verbindung 1 mit der Elektronenbeugungsstudie am 2,2,4,4-Tetrafluor-1,3-diselenetan sowie mit den beiden Röntgenstrukturanalysen des 2,2,4,4-Tetraacetyl-1,3-diselenetans zeigt Tab. IV.

Die für Verbindung 1 gefundenen Werte für die wichtigsten Bindungslängen und -winkel stimmen gut mit den in der Literatur gefundenen überein.

Die Se-C-Bindungslängen in Verbindung $\mathbf{1}$ sind trotz der Ringspannung kürzer als der ent- sprechende Wert in der vergleichbaren aliphatischen Verbindung $\left(\mathrm{CF}_{3}\right)_{2} \mathrm{Se}(198,0(9) \mathrm{pm})$ [10]. Mit diesem kurzen $\mathrm{Se}-\mathrm{C}$-Abstand geht ein relativ kurzer Se'.Se-Kontakt (293,1 pm) einher, der eher im Bereich der kovalenten Bindung (234 pm) [11] als des Van der Waals' Abstands (400 pm) [12] liegt. Die starke bindende Wechselwirkung zwischen den Se-Atomen resultiert vermutlich im wesentlichen aus der Überlappung von Hybridorbitalen. Diese bindende Wechselwirkung dürfte verantwortlich sein für die beobachtete Verkürzung 
der $\mathrm{Se}-\mathrm{C}$-Bindungslängen in 1. Ein Vergleich der ${ }^{77} \mathrm{Se}-\mathrm{NMR}$-Daten von fluor- bzw. trifluormethylsubstituierten 1,3-Diselenetanen ergibt eine interessante Wechselbeziehung zwischen der Anzahl der Fluoratome bzw. der Trifluormethylgruppen am 1,3-Diselenetangerüst und der ${ }^{77} \mathrm{Se}$-Verschiebung (Tab. V).

Tab. V. ${ }^{77} \mathrm{Se}-\mathrm{NMR}-$ Verschiebungen ausgewählter 1,3Diselenetane.

\begin{tabular}{llc}
\hline & & $\delta(\mathrm{Se})(\mathrm{ppm})^{*}$ \\
\hline $\mathbf{1}$ & & 667,6 \\
cis $-\left(\mathrm{CF}_{3}\right)_{2} \mathrm{~F}_{2} \mathrm{C}_{2} \mathrm{Se}_{2}$ & {$[13]$} & 874,6 \\
trans $-\left(\mathrm{CF}_{3}\right)_{2} \mathrm{~F}_{2} \mathrm{C}_{2} \mathrm{Se}_{2}$ & {$[13]$} & 875,3 \\
$\mathrm{~F}_{4} \mathrm{C}_{2} \mathrm{Se}_{2}$ & {$[7]$} & $1149,3 * *$ \\
\hline
\end{tabular}

* Externer Standard ist $\left(\mathrm{CH}_{3}\right)_{2} \mathrm{Se}$;

** der in der Literatur angegebene Wert ist bezogen auf $\mathrm{SeOCl}_{2}$ als Standard. Dieser wurde umgerechnet auf $\left(\mathrm{CH}_{3}\right)_{2} \mathrm{Se}$.
Wie man Tab. V entnehmen kann, nimmt mit fortschreitendem Ersatz der Trifluormethylgruppen am 1,3-Diselenetangerüst durch Fluoratome die ${ }^{77} \mathrm{Se}$-Verschiebung in etwa linear zu.

Verbindung 1 ist ein interessantes Edukt für die Synthese trifluormethylsubstituierter selenhaltiger Heterocyclen und läßt eine breite Folgechemie erwarten.

Wir danken dem Fonds der Chemischen Industrie, der Deutschen Forschungsgemeinschaft, der Nato und der VW-Stiftung für finanzielle Unterstützung. Der Hoechst AG danken wir für die Bereitstellung von Chemikalien. G. R. dankt dem Fonds der Chemischen Industrie für die Vergabe eines Promotionsstipendiums.
[1] M. Witt, K. S. Dhathathreyan und H. W. Roesky, Adv. Inorg. Chem. Radiochem. 30, 223 (1986).

[2] H. W. Roesky und K. S. Dhathathreyan, J. Chem. Soc., Chem. Commun. 1984, 1053; H. W. Roesky, K. S. Dhathathreyan, M. Noltemeyer und G. M. Sheldrick, Z. Naturforsch. 40 b, 240 (1985); K. Burger und E. Huber, Chem.-Ztg. 110, 211 (1986); H. Grützmacher, N. Keweloh, H. W. Roesky, M. Noltemeyer und G. M. Sheldrick, J. Fluorine Chem. 37, 279 (1987); K. Burger und R. Simmerl, Liebigs Ann. Chem. 1984, 982; A. Elsäßer und W. Sundermeyer, Tetrahedron Lett. 24, 2141 (1983); A. Elsäßer und W. Sundermeyer, Chem. Ber. 118, 4553 (1985); G. Rabe, J. Sundermeyer, H. W. Roesky, H.-G. Schmidt und M. Noltemeyer, Chem. Ber. 123, 691 (1990).

[3] D. C. England, J. Org. Chem. 46, 153 (1981); M. V. D. Puy und L. G. Agnello, J. Org. Chem. 47, 377 (1982).

[4] Y. L. Kopaevich, G. G. Belen'kii, E. I. Mysov, L. S. German und I. L. Knunyants, Zh. Vses. Khim. Obshchest. 17, 226 (1972); C. A. 77, 101427 q (1972).
[5] M. S. Raasch, J. Org. Chem. 45, 3517 (1980).

[6] Weitere Einzelheiten zur Kristallstrukturuntersuchung können beim Fachinformationszentrum Karlsruhe, Gesellschaft für wissenschaftlich-technische Information $\mathrm{mbH}, \mathrm{D}-7514$ Eggenstein-Leopoldshafen 2, unter Angabe der Hinterlegungsnummer CSD 54793, des Autors und des Zeitschriftenzitats angefordert werden.

[7] T. Wehrung, H. Oberhammer, A. Haas, B. Koch und N. Welcman, J. Mol. Struct. 35, 253 (1976).

[8] R. Kivekäs, T. Laitalainen und T. Simonen, Acta Chem. Scand. B 40, 98 (1986).

[9] R. J. Adrien, R. W. Gable, B. F. Hoskins und D. Dakternieks, J. Org. Chem. 359, 33 (1989).

[10] C. J. Marsden und G. M. Sheldrick, J. Mol. Struct. 10, 405 (1971).

[11] L. Pauling, Die Natur der Chemischen Bindung, S. 218, Verlag Chemie, Weinheim (1973).

[12] L. Pauling, Die Natur der Chemischen Bindung, S. 249, Verlag Chemie, Weinheim (1973).

[13] A. Haas und M. Spehr, Chimia 42, 265 (1988). 